ELECTRONIC RESEARCH ANNOUNCEMENTS OF THE AMERICAN MATHEMATICAL SOCIETY

Volume 4, Pages 1-3 (February 13, 1998)

S $1079-6762(98) 00039-0$

\title{
SOME LIE RINGS ASSOCIATED WITH BURNSIDE GROUPS
}

\author{
M. F. NEWMAN AND MICHAEL VAUGHAN-LEE
}

(Communicated by Efim Zelmanov)

\begin{abstract}
We describe some calculations in graded Lie rings which provide a fairly sharp upper bound for the nilpotency class and for the order of the restricted Burnside group on two generators with exponent 7 .
\end{abstract}

The purpose of this announcement is to report on some, machine-based, calculations in graded Lie rings which provide a fairly sharp upper bound for the nilpotency class and for the order of the restricted Burnside group $R(2,7)$ on two generators with exponent 7 .

The associated Lie ring of $R(2,7)$ may be viewed as a Lie algebra over the field $\mathbb{Z}_{7}$ with 7 elements. Furthermore, it is known to satisfy certain multilinear identities, the most significant of which is equivalent to the 6-Engel identity. Additional multilinear identities are described in [3] (Section 2.5). We let $E(2,7)$ be the largest two-generator Lie algebra over $\mathbb{Z}_{7}$ satisfying the 6 -Engel identity, and let $W(2,7)$ be the largest two-generator Lie algebra over $\mathbb{Z}_{7}$ satisfying all the multilinear identities described in [3]. So the associated Lie ring of $R(2,7)$ is a homomorphic image of $W(2,7)$, which is itself a homomorphic image of $E(2,7)$.

Earlier calculations, described in [1], showed that the largest class 18 quotient of $E(2,7)$ has dimension 6473 and the largest class 18 quotient of $W(2,7)$ has dimension 6366 . This result required the use of a multilinear identity of degree 13 first pointed out by Wall [6]. This, together with results of Wall [7], implies that the largest class 18 quotient of $R(2,7)$ has order $7^{6366}$.

At that time Havas et al. [1] made the guess that the class of $W(2,7)$ is at least 30 and its dimension is at least 20000. (In [3] a more conservative estimate is given: the order of $R(2,7)$ is at least $7^{10000}$.)

A rather astronomical upper bound for the class of $R(m, 7)$ was obtained by Vaughan-Lee and Zelmanov [5] (Theorem 4). More recently Vaughan-Lee [4] has obtained the reasonably moderate upper bound $51 \mathrm{~m}^{8}$ for the class of $R(m, 7)$. Moreover, these methods, augmented by appropriate computations, can be pushed quite a bit further to give bounds in the hundreds for the class of $R(2,7)$.

With recent improvements in computer hardware and the program, it seemed worth making further calculations in the spirit of [1]. For example, the program now allows closure under automorphisms - as described in [2] for the group case. With some additional refinements - in particular, the use of a more compact data structure suggested by George Havas - it has turned out to be possible to show that $E(2,7)$ has class 29 and dimension 23789.

Received by the editors November 20, 1997.

1991 Mathematics Subject Classification. Primary 17-04, 17B30, 20 D15.

(C)1998 American Mathematical Society 
Showing these are upper bounds turns out to require only moderate resources, at most $120 \mathrm{MB}$ and about 30 hours on a Dec Alpha. To confirm that the product presentation obtained this way is consistent and that the algebra so defined satisfies the 6-Engel condition is, of course, much more demanding. It takes about 200 hours depending on the machine used (in our case various machines).

Moreover, we have taken the next step. First enforce instances of Wall's degree 13 multilinear identity in addition to the 6-Engel identity. This gives an upper bound of 20418 for the dimension of $W(2,7)$. The product presentation so defined has class 29 and can be stored in about $90 \mathrm{MB}$. Confirming that this algebra satisfies Wall's multilinear identity requires further lengthy computations. It then only needs a few computations to show that it satisfies all the multilinear identities of $R(2,7)$ and hence that $W(2,7)$ does indeed have dimension 20418 and class 29.

These calculations show that $R(2,7)$ has order at most $7^{20418}$. It seems likely that the actual order will be close to this bound.

We have begun the lengthy task of determining the order of $R(2,7)$.

The ranks of the homogeneous components from weight 18 are given in the table (the ranks for lower weights are given in [3], Section 7.3).

\begin{tabular}{|r|r|r|r|r|}
\hline & \multicolumn{2}{|c|}{$E(2,7)$} & \multicolumn{2}{c|}{$W(2,7)$} \\
\hline Class & Rank & Total & Rank & Total \\
\hline 18 & 2221 & 6473 & 2157 & 6366 \\
19 & 3136 & 9609 & 2992 & 9358 \\
20 & 4104 & 13713 & 3795 & 13153 \\
21 & 4716 & 18429 & 4046 & 17199 \\
22 & 4039 & 22468 & 2850 & 20049 \\
23 & 1192 & 23660 & 240 & 20289 \\
24 & 96 & 23756 & 96 & 20385 \\
25 & 14 & 23770 & 14 & 20399 \\
26 & 14 & 23784 & 14 & 20413 \\
27 & 2 & 23786 & 2 & 20415 \\
28 & 1 & 23787 & 1 & 20416 \\
29 & 2 & 23789 & 2 & 20418 \\
\hline
\end{tabular}

Let $E[i, j]$ be the multiweight component of $E(2,7)$ spanned by the Lie products of weight $i$ in the first generator and weight $j$ in the second generator. The calculations show that the multiweight components $E[i, j]$ with $i \geq 16$ are trivial. This suggests that perhaps the normal closure of an element in $R(m, 7)$ has class 15 and so the class of $R(m, 7)$ is at most $15 \mathrm{~m}$. Calculations to settle this seem to be out of reach at present.

\section{ACKNOWLEDGEMENTS}

This work was partly supported by grants for visits from the Mathematical Sciences Research Visitor Program of the Australian National University and the Engineering and Physical Sciences Research Council (EPSRC) of the United Kingdom (grant number GR/L36079), and by grants for computing from EPSRC (grant number GR/L60326) and the ANU Supercomputing Facility. 


\section{REFERENCES}

[1] George Havas, M. F. Newman, and M. R. Vaughan-Lee, A nilpotent quotient algorithm for graded Lie rings, J. Symbolic Comput. 9 (1990), 653-664. MR 92d:20054

[2] M. F. Newman and E. A. O'Brien, Application of computers to questions like those of Burnside. II, Internat. J. Algebra Comput. 6 (1996), 593-605. MR 97k:20002

[3] Michael Vaughan-Lee, The restricted Burnside problem, London Mathematical Society Monographs, New Series, vol. 8, 2nd ed., Clarendon Press, Oxford, 1993. MR 98b:20047

[4] Michael Vaughan-Lee, The nilpotency class of finite groups of exponent $p$, Trans. Amer. Math. Soc. 346 (1994), 617-640. MR 95g:20021

[5] Michael Vaughan-Lee and E. I. Zelmanov, Upper bounds in the restricted Burnside problem, J. Algebra 162 (1993), 107-145. MR 94j:20019

[6] G. E. Wall, On the Lie ring of a group of prime exponent, Proc. Second Internat. Conf. Theory of Groups, Canberra, 1973, pp. 667-690, Lecture Notes in Math., vol. 372, SpringerVerlag, Berlin, Heidelberg, New York, 1974. MR 50:10098

[7] G. E. Wall, On the Lie ring of a group of prime exponent. II, Bull. Austral. Math. Soc. 19 (1978), 11-28. MR 80b:20052

Australian National University

E-mail address: newman@maths.anu.edu.au

UNIVERSITY OF OXFORD

E-mail address: vlee@maths.oxford.ac.uk 